\title{
Eficiência bioeconômica da adubação de pastagem natural no sul do Brasil
}

\author{
Bioeconomic efficiency of native pasture fertilization in southern Brazilian
}

\author{
Davi Teixeira dos Santos ${ }^{\mathrm{I}^{*}}$ Paulo César de Faccio Carvalho" ${ }^{\mathrm{II}}$ Carlos Nabinger ${ }^{\mathrm{II}}$ \\ Igor Justin Carassai' ${ }^{I}$ Luiz Hipólito Gomes ${ }^{\text {III }}$
}

\section{RESUMO}

O objetivo deste trabalho foi avaliar as produções vegetal e animal e a resposta econômica de uma pastagem natural submetida à adubação de correção e doses de nitrogênio durante a estação de crescimento 1998/99. O experimento foi conduzido em área da Estação Experimental Agronômica da Universidade Federal do Rio Grande do Sul (EEA - UFRGS). Em 1996 foram aplicados $3000 \mathrm{~kg} \mathrm{ha}^{-1}$ de calcário e adubação correspondente a $500 \mathrm{~kg} \mathrm{ha}^{-1}$ da fórmula 5-20-20. Na análise econômica, considerou-se um terço destas quantidades como referente à utilização na estação de crescimento 1998/99. Os tratamentos foram doses de nitrogênio (N) na forma de uréia (zero, 100 e $200 \mathrm{~kg} \mathrm{ha}^{-1} \mathrm{de} \mathrm{N}$ ), em um delineamento de blocos ao acaso com duas repetições de área por tratamento. Os animais experimentais foram novilhas de corte com um ano de idade e peso médio inicial de $160 \mathrm{~kg}$. A massa de forragem, a taxa de acúmulo diário de matéria seca, a produção total de matéria seca verde e a eficiência de transformação do $\mathrm{N}$ em produção primária foram maiores $(P<0,1)$ quanto maior foi a dose de $N$ aplicado. As aplicações de 100 e $200 \mathrm{~kg} \mathrm{ha}^{-1}$ de $\mathrm{N}$ elevaram os custos em 104,1 e $208,2 \%$, respectivamente. A aplicação de $200 \mathrm{~kg} \mathrm{ha}^{-1}$ de $\mathrm{N}$ em pastagem natural apresentou maior produção de peso vivo, margem bruta e eficiência de conversão do $\mathrm{N}$ em produto animal. Quando foram utilizados apenas o calcário e a adubação $N-P-K$ de correção, obteve-se o maior retorno financeiro direto. A pastagem natural mostrou resposta positiva à aplicação de fertilizantes, dentro da amplitude de quantidade destes insumos aplicadas no presente trabalho.

Palavras-chave: Campos Sulinos, eficiência de utilização do nitrogênio, margem bruta, nitrogênio, produção animal.

\section{ABSTRACT}

This research was aimed at evaluating the plant and animal production and the economic response of a native

\begin{abstract}
pasture submitted to the application of $N-P-K$ and nitrogen levels during the 1998/99 growing season. The experiment was conducted at Estação Experimental Agronômica of the Universidade Federal do Rio Grande do Sul (EEA - UFRGS). The pasture was fertilized in 1996 using $3000 \mathrm{~kg} \mathrm{ha}^{-1}$ of lime and $500 \mathrm{~kg} \mathrm{ha}^{-1}$ of 5-20-20. The one third of these amounts was considered as that concerning the 1998/99 growing season, in the economic analysis. The treatments were nitrogen $(N)$ levels (zero, 100 and $200 \mathrm{~kg} \mathrm{ha}^{-1}$ of N) as urea, in a randomized block design with two plots by treatment. The experimental animals were beef heifers with initial age of 1,5 year-old and average live weight of $160 \mathrm{~kg}$. The forage mass, dry matter accumulation rate, green dry matter total production and the $N$ transformation efficiency in primary production were enhanced by the $N$ level applied $(P<0.1)$. The treatments 100 and $200 \mathrm{~kg} \mathrm{ha}^{-1}$ of $N$ increased the costs in 104.1 and $208.2 \%$, respectively. The treatment $200 \mathrm{~kg} \mathrm{ha}^{-1}$ of $N$ presented the greater area live weight gain, the gross margin and the $N$ conversion efficiency in animal product. The greater net financial return was reached when only lime and $N-P-K$ fertilization were applied. The native pasture showed a positive response to the fertilizer application, within the amplitude of amount of the input applied in this experiment.
\end{abstract}

Key words: Campos Sulinos, nitrogen use efficiency, gross margin, nitrogen, animal production.

\section{INTRODUÇÃO}

O bioma Campos Sulinos constitui-se num dos mais importantes patrimônios ecológicos da região Sul do Brasil, extendendo-se pelo Uruguai, leste da Argentina e sul do Paraguai. Campos refere-se a um tipo de vegetação em que predominam gramíneas e

\footnotetext{
IPrograma de Pós-graduação em Zootecnia/Plantas Forrageiras, Universidade Federal do Rio Grande do Sul (UFRGS). Av. Bento Gonçalves, 7712, 91540-000, Porto Alegre, RS, Brasil. E-mail: daviteixeira@hotmail.com. *Autor para correspondência.

IIDepartamento de Plantas Forrageiras e Agrometeorologia, Faculdade de Agronomia, UFRGS, Porto Alegre, RS, Brasil.

IIIAutônomo.
} 
outras herbáceas. As áreas de pastagens componentes deste ecossistema representam o principal recurso forrageiro utilizado na alimentação dos rebanhos nas regiões de abrangência desta vegetação (BERRETA et al., 2000; NABINGER et al., 2000). A despeito de sua importância, a utilização de pastagens naturais para criação de bovinos de corte no sul do país vem, de longa data, sendo praticada de maneira abusiva, de forma a não permitir a estabilização do sistema pecuário em um patamar de produtividade condizente com seu potencial. Os excessos de lotação animal e a não reposição dos nutrientes extraídos do ecossistema constituem as principais causas do esgotamento dos campos e, conseqüentemente, da redução de sua capacidade de suporte e potencial produtivo ao longo dos anos.

No Rio Grande do Sul (RS), o aumento das áreas de silvicultura e lavoura, sobretudo em momentos de valorização de produtos agrícolas, vem promovendo drástica redução das áreas destinadas à exploração pecuária, o que gera uma demanda por alternativas que promovam a sustentabilidade bioeconômica do sistema. Nos últimos 25 anos, a área de pastagens naturais sofreu uma redução de $27,6 \%$, de 14,5 para 10,5 milhões de hectares (NABINGER et al., 1999), ao passo que o rebanho bovino, no mesmo período, aumentou em mais de $50 \%$, de 8,5 para 13,2 milhões de cabeças (ANUALPEC, 2004). Tais números deflagram a falta de cuidado com que produtores e autoridades ligadas ao setor vêm tratando esta questão.

Às instituições de pesquisa cabe alertar as autoridades competentes e oferecer aos produtores alternativas de manejo que visem à manutenção do bioma Campos Sulinos, com vistas a assegurar a preservação ambiental e aumentar a eficiência de utilização deste recurso. A reposição de nutrientes ao solo e sua eficiente reciclagem pode ser peça-chave neste processo. Alguns resultados de pesquisa indicam que a pastagem natural pode apresentar resposta tão positiva à adubação quanto qualquer outro tipo de pastagem. A fertilização nitrogenada proporciona aumentos nos níveis de produção de forragem, qualidade e distribuição estacional da forragem (BARCELLOS et al., 1987; BEMHAJA et al., 1998; BOGGIANO et al., 2000; GUMA, 2005).

O uso de fertilizantes, todavia, é a ferramenta tecnológica mais questionada por produtores rurais quanto à sua viabilidade econômica. Nesse sentido, o presente trabalho teve como proposta avaliar, em uma pastagem natural submetida à adubação de correção e a doses de nitrogênio $(\mathrm{N})$, a produção de forragem e de peso vivo, bem como a eficiência de conversão do $\mathrm{N}$ em produto animal e a resposta financeira, gerada a partir dos resultados de produção de peso vivo por unidade de área. Tais objetivos estão fundamentados na hipótese de que a produção de biomassa aérea da pastagem natural é limitada pela deficiência de nutrientes no solo. Mmesmo que esta condição fosse melhorada com a aplicação de corretivos e adubação de manutenção, a disponibilidade de $\mathrm{N}$ continuaria a ser um fator limitante à expressão do potencial produtivo da pastagem natural.

\section{MATERIAL E MÉTODOS}

O experimento foi conduzido em área de pastagem natural pertencente à Estação Experimental Agronômica da Universidade Federal do Rio Grande do Sul (EEA - UFRGS), situada no km 146 da BR - 290, município de Eldorado do Sul - RS (3005'52” S, 5139'08” W e altitude média de 46 metros), na região fisiográfica denominada de Depressão Central. O clima da região é Cfa (subtropical úmido), segundo a classificação de Köppen (MORENO, 1961). O solo da área experimental é um Argissolo Vermelho Distrófico, que tem como característica um solo profundo, bem drenado, e de textura arenosa a franco argilosa (EMBRAPA, 1999). A vegetação é composta predominantemente de Paspalum notatum e outras gramíneas, com menor participação de leguminosas (Desmodium incanum) e espécies indesejáveis (Eryngium horridum e Vernonia nudiflora).

O experimento foi realizado de 20 de outubro de 1998 a 10 de junho de 1999, quando foram testadas três doses de nitrogênio (N) em cobertura (zero, 100 e $200 \mathrm{~kg} \mathrm{ha}^{-1}$ de $\mathrm{N}$, na forma de uréia), as quais constituíram os tratamentos. A adubação nitrogenada nos tratamentos 100 e $200 \mathrm{~kg} \mathrm{ha}^{-1}$ de $\mathrm{N}$ foi parcelada em duas aplicações iguais, em 20 de setembro de 1998 e 12 de janeiro de 1999. Em 1996 a área experimental recebeu adubação de correção/manutenção, com aplicação de $3000 \mathrm{~kg} \mathrm{ha}^{-1}$ de calcário e adubação correspondente a $500 \mathrm{~kg} \mathrm{ha}^{-1}$ da fórmula 5-20-20. Para fins de análise econômica, a referida adubação foi amortizada em três anos, utilizando-se, portanto, um terço das quantidades aplicadas para o estudo da resposta financeira de 1998/ 99. Os animais experimentais foram novilhas mestiças de corte com um ano de idade e peso médio inicial de $160 \mathrm{~kg}$. Utilizou-se o método de lotação contínua com carga variável, por meio da técnica "put-and-take" (MOTT \& LUCAS, 1952). A oferta diária de forragem (OF) pretendida foi de $9 \%$ (9kg de matéria seca verde [MSV] $100 \mathrm{~kg}^{-1}$ de peso vivo [PV]) em todos os tratamentos. Os animais foram pesados e a carga animal ajustada a cada 28 dias.

A massa de forragem (MF, em $\mathrm{kg} \mathrm{ha}^{-1}$ de MS) foi estimada a partir de leituras da altura 
comprimida do pasto com disco medidor de forragem. Foram realizadas 100 leituras por unidade experimental, a cada 28 dias. O valor médio da altura do pasto foi utilizado como variável independente em equações do tipo $\mathrm{Y}=\mathrm{a}+\mathrm{bX}$, que relacionam a altura do pasto com a MF. Para a calibração do disco medidor, em cada avaliação foram escolhidas 10 amostras de distintas alturas em cada unidade experimental, as quais foram cortadas rente ao solo e posteriormente separadas manualmente em material verde e material morto (MM, em \%). Para a MF foi desconsiderada a fração MM. A altura média do pasto (ALT, em $\mathrm{cm}$ ) foi estimada pelo disco medidor de forragem, em que cada unidade de disco correspondeu a $0,5 \mathrm{~cm}$. Para a estimativa da taxa de acúmulo de matéria seca (TAC, em kg ha-1 dia $^{-1}$ ), utilizaram-se três gaiolas de exclusão ao pastejo por unidade experimental, através da metodologia descrita por MORAES et al. (1990). A produção total de matéria seca verde (PMSV, em $\mathrm{kg} \mathrm{ha}^{-1}$ de MSV) foi calculada pela multiplicação dos valores de TAC pelo número de dias do período de avaliação. A oferta real de forragem (OF, em \% do PV) foi obtida multiplicando-se por 100 a disponibilidade de forragem diária média (MF média/ 28 dias + TAC) e dividindo-se o valor obtido pela carga animal média (CA, em kg de $\mathrm{PV} \mathrm{ha}{ }^{-1}$ ) do período de avaliação correspondente.

Para o cálculo da eficiência de utilização do nitrogênio (EUN, em kg MSV kg-1de N), subtraiu-se a PMSV obtida no tratamento controle (Zero $\mathrm{N}$ ) dos resultados obtidos nos tratamentos 100 e 200kg ha-1 de $\mathrm{N}$ e dividiu-se pela quantidade de $\mathrm{N}$ aplicada em cada dose. A produção animal por hectare (GPV, em kg de PV ha-1) foi obtida pelo produto do ganho de peso médio diário (GMD, em kg animal ${ }^{-1}$ ) dos animais avaliadores (três novilhas por unidade experimental) com o número de animais dia ha-1 (produto da taxa de lotação média, em animais ha ${ }^{-1}$ e o número de dias de pastejo). A avaliação econômica foi realizada com a utilização de planilhas de cálculo do software EXCEL versão 5.0, segundo modelo proposto por PÖTTER et al. (2000). Os preços utilizados para o cálculo dos custos totais (CT) e receita bruta (RB) foram atualizados para maio de 2006. Nos componentes do custo envolvendo mão-de-obra e/ou tratores e implementos estão incluídos os gastos com “horas-homem”, com base no salário mínimo rural do RS, e os custos de depreciação e conservação por hora máquina. A RB foi obtida pelo produto do GPV com o valor médio pago pelo kg vivo da novilha na mesma época da avaliação dos custos, que foi de $R$ \$ 1,50. Da relação RB/CT obteve-se o retorno financeiro direto (RFD) por tratamento, representado pelo valor capitalizado para cada $\mathrm{R} \$ 1,00$ investido. A margem bruta (MB) foi obtida pela diferença entre RB e CT. Para a eficiência de conversão do N em produto animal, subtraiu-se o GPV dos tratamentos 100 e $200 \mathrm{~kg} \mathrm{ha}^{-1}$ de $\mathrm{N}$ daquela obtida no tratamento controle (Zero $\mathrm{N}$ ) e dividiu-se pela quantidade de $\mathrm{N}$ aplicada em cobertura.

O delineamento experimental foi o de blocos ao acaso, com duas repetições de área por tratamento. Os resultados foram submetidos à análise de variância e teste $\mathrm{F}$, e quando detectadas diferenças aplicou-se o teste Tukey a 10\% de significância, utilizando-se o software SAS versão 6.0. Os resultados de PMSV, GPV e MB foram submetidos à análise de variância da regressão pelos procedimentos GLM e REG do mesmo software.

\section{RESULTADOS E DISCUSSÃO}

Na tabela 1 encontram-se os resultados de oferta real de forragem (OF), massa de forragem (MF), taxa de acúmulo de matéria seca (TAC), percentagem de material morto (MM) e altura média comprimida do pasto (ALT). A OF ficou um pouco acima da pretendida, porém, foi possível um bom controle desta variável de forma a permitir que fosse semelhante $(\mathrm{P}=0,836)$ entre os tratamentos, sendo na média das três doses igual a $10,9 \%$. Isso respalda a discussão dos resultados de produção animal e de resposta econômica do experimento. Mesmo com a manutenção de OF similares, a MF apresentou resposta linear às doses de $\mathrm{N}(\hat{\mathrm{y}}=$ $\left.1.008,2+2,155 x ; R^{2}=0,312 ; \mathrm{P}=0,059\right)$. Este comportamento da MF foi o mesmo observado na TAC em função das doses de $\mathrm{N}\left(\hat{\mathrm{y}}=7,611+0,0342 \mathrm{x} ; \mathrm{R}^{2}=\right.$ $0,371 ; \mathrm{P}=0,035)$. Isso pressupõe que, mesmo partindose de uma massa inicial semelhante entre os tratamentos $\left(1.704 \mathrm{~kg} \mathrm{ha}^{-1}\right.$ de $\left.\mathrm{MS} ; \mathrm{P}=0,232\right)$ e com o ajuste periódico da carga animal, diferentes ritmos de acúmulo de forragem promovem gradativamente a formação de um gradiente de massa de forragem, e a OF passou a ser controlada em condições distintas de biomassa aérea. A TAC (Tabela 1) apresentou valores abaixo do esperado para este tipo de pastagem, devido ao déficit hídrico que ocorreu durante o período experimental. BERRETA et al. (1998) obtiveram valores de $35 \mathrm{~kg} \mathrm{ha}^{-1} \mathrm{dia}^{-1}$ de MS durante a primavera e de até $61 \mathrm{~kg} \mathrm{ha}^{-1} \mathrm{dia}^{-1}$ de MS no verão, utilizando $92 \mathrm{~kg} \mathrm{ha}^{-1} \mathrm{de}$ N. GUMA (2005), na mesma área experimental e utilizando os mesmos tratamentos do presente estudo, observou TAC de 83,7 kg ha ${ }^{-1}$ dia $^{-1}$ de MS no período de abril a maio, na média das três doses de $\mathrm{N}$.

A altura do pasto (ALT) seguiu a tendência da $\mathrm{MF}$ e foi superior quanto maior a dose de $\mathrm{N}(\hat{\mathrm{y}}=$ $\left.4,179+0,00487 x ; R^{2}=0,395 ; P=0,028\right)$. O ponto mais interessante a destacar com relação a esta variável foi 
Tabela 1 - Oferta real de forragem (OF, \% PV), massa de forragem (MF, $\mathrm{kg} \mathrm{ha}^{-1}$ de MS), taxa de acúmulo de matéria seca (TAC, kg ha $^{-1} \operatorname{dia}^{-1}$ ), material morto da MF total (MM, \%) e altura média do pasto (ALT, $\mathrm{cm}$ ) de uma pastagem natural* submetida a doses de nitrogênio (N). Rio Grande do Sul, Brasil, 1998/99.

\begin{tabular}{llllll}
\hline $\mathrm{N}\left(\mathrm{kg} \mathrm{ha}^{-1}\right)$ & OF & MF & TAC & MM & $3,97 \mathrm{~b}$ \\
\hline Zero & 10,6 & $975,5 \mathrm{~b}$ & $8,1 \mathrm{~b}$ & 36,1 & 35,4 \\
100 & 11,4 & $1.289,0 \mathrm{ab}$ & $10,1 \mathrm{ab}$ & 36,7 & $3,08 \mathrm{a}$ \\
200 & 10,9 & $1.406,5 \mathrm{a}$ & $14,9 \mathrm{a}$ & 36,1 & $5,95 \mathrm{a}$ \\
Média & 10,9 & $1.223,7$ & 11,0 & 3,67 \\
CV (\%) & 16,00 & 19,84 & 32,82 & 0,6434 \\
P & 0,8363 & 0,1000 & 0,0867 & 9,97 \\
\hline
\end{tabular}

Médias seguidas por letras distintas, na coluna, diferem pelo teste de Tukey $(\mathrm{P}<0,1)$.

* Pastagem constituída predominantemente por Paspalum notatum.

o bom grau de relacionamento apresentado com a MF, com coeficiente de correlação de Pearson (r) de $0,7(\mathrm{P}=$ 0,0175). De forma geral, correlação média-alta entre ALT e MF somente é observada em pastagens cultivadas, mais homogêneas. Em pastagens naturais, a variabilidade do ambiente de pastejo - sobretudo a heterogeneidade espacial - normalmente impede a obtenção de boas relações entre estas variáveis. No presente estudo, no entanto, a correlação de $70 \%$ pode ser considerada expressiva e a relação MF/ALT pode servir de parâmetro para outros estudos envolvendo ambas variáveis em pastagem natural. Conforme CARVALHO et al. (2001), a altura, para os animais, significa quantidade de biomassa disponível, e a sua preferência por altura representa a oportunidade de alta ingestão na medida em que esta potencializa a profundidade e, conseqüentemente, a massa do bocado. Além disso, do ponto de vista da transmissão de tecnologia, a altura do pasto como critério de manejo é de mais fácil entendimento por parte do produtor e, portanto, deve ser explorada pela pesquisa científica quanto a suas relações com as respostas primária e secundária (SANTOS et al., 2003). Os valores obtidos da relação MF/ALT foram de 246; 253 e 284kg ha-1 de MSV para cada cm na altura do pasto, respectivamente para as doses zero, 100 e $200 \mathrm{~kg} \mathrm{ha}^{-1}$ de $\mathrm{N}(\mathrm{P}=0,4549)$.

A figura 1 apresenta uma função resposta linear da pastagem natural em produção de forragem em relação às doses de $\mathrm{N}$ aplicadas. Observando-se a magnitude das respostas, conclui-se que não foi possível verificar o potencial de resposta da pastagem em relação à adubação nitrogenada. As produções de forragem obtidas foram relativamente baixas em todos os tratamentos, em virtude de um déficit hídrico rigoroso ocorrido no período de verão/outono. Em condições hídricas não limitantes e com o uso de

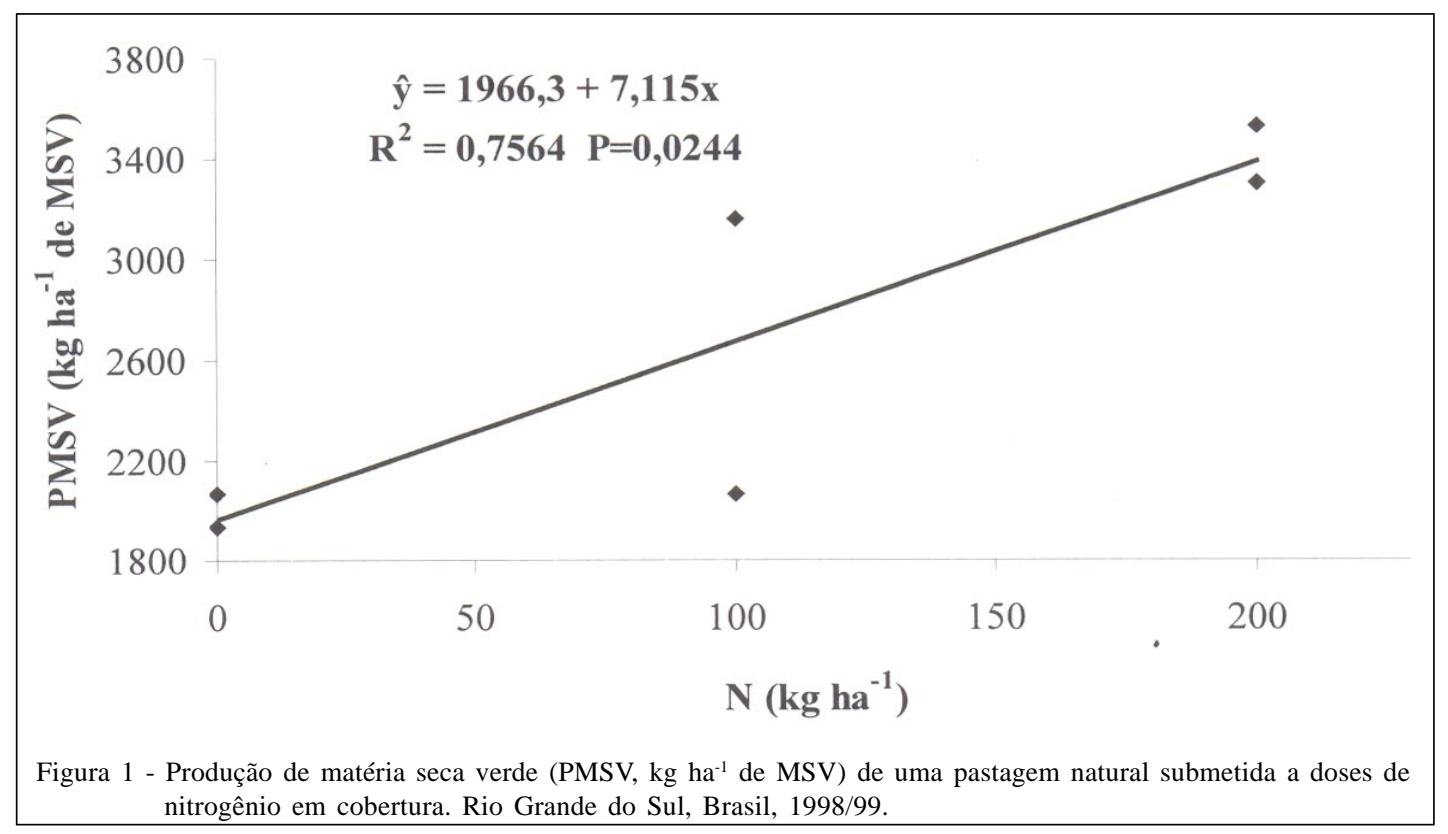

Ciência Rural, v.38, n.2, mar-abr, 2008. 
adubação, MARASCHIN (2000) relatou valores de produção de MS total em pastagem natural de até 12ton ha $^{-1}$. Com relação à eficiência de utilização do nitrogênio (EUN), a transformação deste insumo em produção primária se manteve praticamente constante, com valores de 6,1 e 7,1kg de MSV adicional (em relação ao tratamento Zero N) para cada kg de $\mathrm{N}$ aplicado, nas doses de 100 e $200 \mathrm{~kg} \mathrm{ha}^{-1}$ de $\mathrm{N}$, respectivamente. Este resultado demonstra que a maior dose testada (200kg ha ${ }^{-1}$ de N) ainda está aquém da capacidade de resposta deste tipo de pastagem com fertilização nitrogenada, pois com o aumento do rendimento de matéria seca para cada unidade de nutriente disponível, a EUN decresce à medida que o rendimento se aproxima do potencial máximo de produção da planta (MAZZANTI et al., 1994; WHITEHEAD, 1995). AYALA \& CARÁMBULA (1994) relatam valores de EUN para o inverno e verão de 1,5 e 14kg de MS kg-1 de N aplicado, respectivamente.

A figura 2 apresenta as tendências de comportamento produtivo (a) e econômico (b) da pastagem natural frente à aplicação de fertilizantes, mais precisamente no que se refere às doses de $\mathrm{N}$ em cobertura. A carga animal suportada pela pastagem aumentou $(\mathrm{P}<0,1)$, conforme a dose de $\mathrm{N}$, sendo os valores médios de 572; 752 e $854 \mathrm{~kg} \mathrm{ha}^{-1}$ de PV, respectivamente para as doses zero, 100 e $200 \mathrm{~kg} \mathrm{ha}^{-1}$ de $\mathrm{N}$. O ganho de peso médio diário não diferiu entre os tratamentos $(\mathrm{P}>0,1)$ e foi, na mesma ordem, de 0,42 ; 0,35 e $0,44 \mathrm{~kg}_{\text {animal }}{ }^{-1}$ dia $^{-1}$. Em termos de produção animal por área (Figura 2a), a resposta da pastagem à aplicação de $\mathrm{N}$ foi bastante moderada até $100 \mathrm{~kg} \mathrm{ha}^{-1}$, em relação àquela verificada em virtude do aumento de 100 para $200 \mathrm{~kg} \mathrm{ha}^{-1}$ de N. Esta não é uma resposta comum no que diz respeito à adubação nitrogenada. Normalmente, o incremento de produtividade em níveis menores de nitrogênio se dá, em um primeiro momento, de forma linear e positiva. No presente trabalho, no entanto, além da maior inclinação da curva de incremento no GPV a partir de $100 \mathrm{~kg} \mathrm{ha}^{-1}$ de N, foi com a aplicação de $200 \mathrm{~kg} \mathrm{ha}^{-1}$ de $\mathrm{N}$ que se observou a maior eficiência de conversão deste insumo em produto animal, sendo obtida uma taxa de $1,7 \mathrm{~kg}$ de PV adicional (em relação ao tratamento Zero $\mathrm{N}$ ) produzido para cada kg de $\mathrm{N}$ aplicado. Quando se utilizou $100 \mathrm{~kg} \mathrm{ha}^{-1} \mathrm{de} \mathrm{N}$, a taxa de conversão foi de apenas $0,48 \mathrm{~kg}^{\mathrm{de}} \mathrm{PV} \mathrm{kg}^{-1} \mathrm{de}$ N. Não foi possível verificar o potencial de resposta em produção secundária da pastagem natural à aplicação de $\mathrm{N}$, sugerindo-se que outros níveis sejam incluídos neste estudo para melhor detalhamento da função resposta e verificação do ótimo produtivo. GILLEN \& BERG (1998), trabalhando com doses de N em pastagem natural de estação quente, verificaram taxas de conversão da ordem de $2,7 \mathrm{~kg}$ de $\mathrm{PV} \mathrm{kg}^{-1}$ de $\mathrm{N}$.

Cabe ressaltar, ainda com relação à figura

2(a), a excelente resposta do tratamento controle (Zero $\mathrm{N}$ ), em que foram produzidos $363 \mathrm{~kg} \mathrm{ha}^{-1} \mathrm{de} \mathrm{PV}$, o que significa, aproximadamente, quatro vezes a produção média do RS na estação de crescimento da pastagem natural, que é entre 70 e $110 \mathrm{~kg} \mathrm{ha}^{-1}$ de PV. Esta adubação de correção poderia ter como objetivo inicial a recuperação da capacidade de suporte potencial dos campos, em muitos casos comprometida pela extração contínua de nutrientes, sem que haja reposição.

$\mathrm{O}$ incremento apenas moderado na produção animal de zero a $100 \mathrm{~kg} \mathrm{ha}^{-1}$ de $\mathrm{N}$ teve reflexo direto no resultado final da análise econômica do experimento. Na figura 2(b) fica evidenciado o decréscimo na margem bruta $(M B)$ até quantidades de $\mathrm{N}$ próximas a $100 \mathrm{~kg} \mathrm{ha}^{-1}$. Observando-se a linha de tendência, somente a partir de doses de $\mathrm{N}$ próximas a $200 \mathrm{~kg} \mathrm{ha}^{-1}$ é que o investimento feito neste insumo seria compensador em termos de $\mathrm{MB}$ ha ${ }^{-1}$, em relação à correção da fertilidade do solo apenas com calcário e adubação N-P-K de base, sem dose adicional de N em cobertura.

A tabela 2 apresenta uma síntese da análise econômica dos tratamentos. O investimento em $\mathrm{N}$ representou aumentos de $104,1 \%$ e $208,2 \%$ no Custo Total (CT) dos tratamentos em que se aplicou 100 e $200 \mathrm{~kg} \mathrm{ha}^{-1}$ de $\mathrm{N}$, respectivamente, em relação ao tratamento controle (Zero $\mathrm{N}$ ). Na última década, o custo dos fertilizantes elevou-se em proporções muito superiores às variações ocorridas no preço pago ao produtor pelos produtos agropecuários, sobretudo na produção de bovinos de corte (SANTOS et al., 2002). Ainda assim, no presente trabalho, todos os tratamentos responderam de forma positiva sob o ponto de vista econômico, ratificando a hipótese de que a pastagem natural apresenta elevado potencial de resposta à prática da adubação. $\mathrm{O}$ tratamento com aplicação de $200 \mathrm{~kg} \mathrm{ha}^{-1}$ de $\mathrm{N}$ apresentou Receita Bruta (RB) superior aos demais $(\mathrm{P}=0,0074)$, fruto da maior produção de peso vivo por área. A RB semelhante entre as doses zero e $100 \mathrm{~kg} \mathrm{ha}^{-1}$ de $\mathrm{N}$ ocasionou, nesta última, uma margem bruta inferior em função do custo total mais elevado. Com isto, a MB da dose $200 \mathrm{~kg} \mathrm{ha}^{-1} \mathrm{de} \mathrm{N}$ foi superior $(\mathrm{P}=0,0636)$ à dose $100 \mathrm{~kg} \mathrm{ha}^{-1}$ de $\mathrm{N}$, enquanto que o tratamento controle (zero) ficou em posição intermediária, não diferindo das demais. Em última análise, a aplicação de calcário ea adubação NP-K de correção, sem nitrogênio adicional em cobertura, proporcionou maior retorno financeiro direto (RFD) do investimento $(\mathrm{P}=0,0054)$, com a capitalização de $\mathrm{R} \$$ 3,12 para cada $\mathrm{R} \$ 1,00$ desembolsado, superando as 

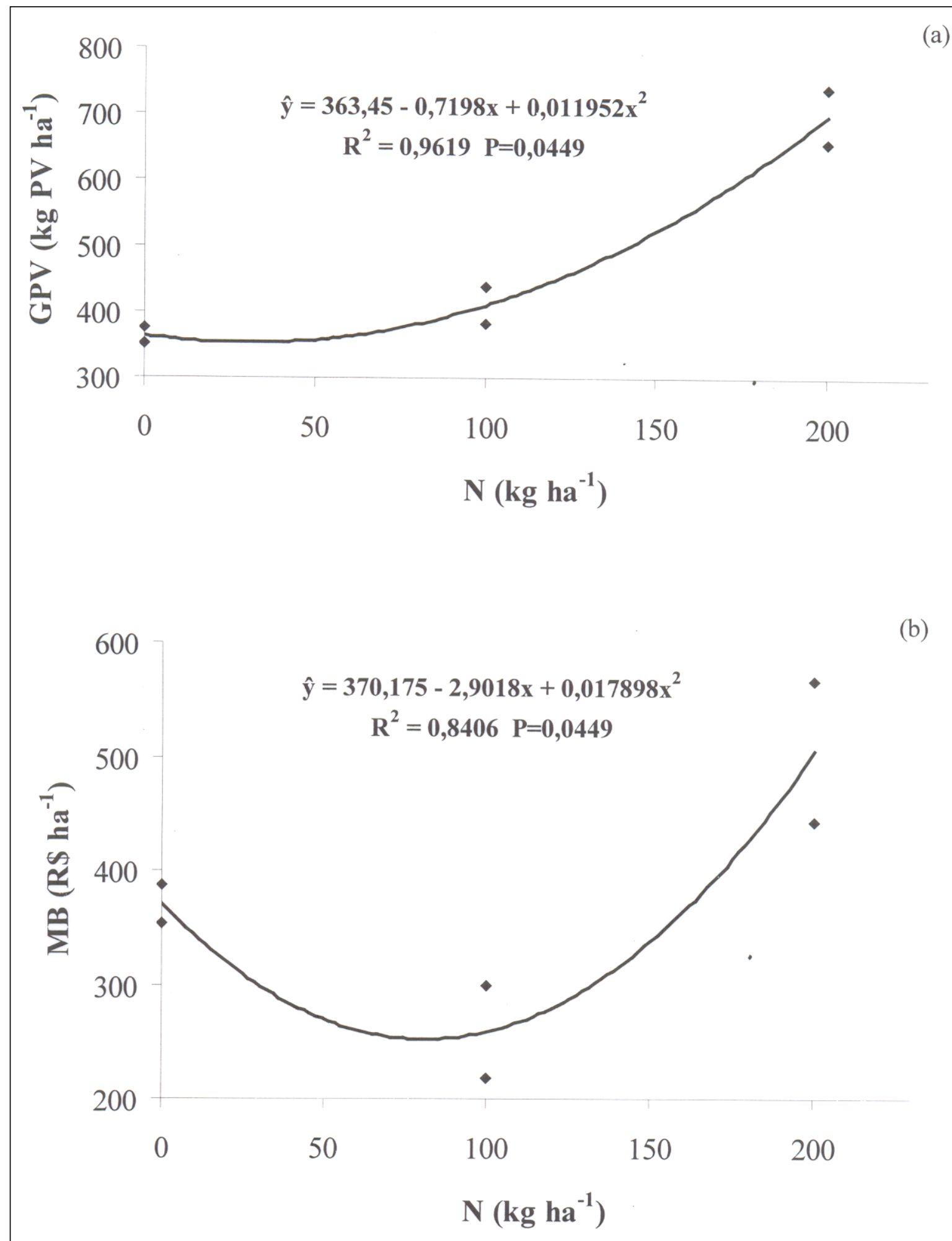

Figura 2 - Estimação da resposta, em produção animal (GPV, kg PV ha-1) (a) e margem bruta por hectare (MB, R\$ ha $\left.{ }^{-1}\right)$ (b), de uma pastagem natural submetida à adubação de correção e doses de nitrogênio em cobertura. Rio Grande do Sul, Brasil, 1998/99. 
Tabela 2 - Resumo da análise econômica da adubação de uma pastagem natural no Rio Grande do Sul, Brasil. Valores atualizados para maio de 2006, em R\$ ha ${ }^{-1}$.

\begin{tabular}{llll}
\hline & & $\mathrm{kg} \mathrm{ha}^{-1}$ de N \\
\cline { 2 - 4 } Componente da análise & Zero & 100 & 65,00 \\
\hline Calcário & 65,00 & 65,00 & 110,00 \\
Adubação correção & 110,00 & 110,00 & 364,44 \\
Adubação nitrogenada & ----- & 182,22 & 539,44 \\
Custo total & 175,00 & 357,22 & $1.045,20$ a \\
Receita bruta & $545,18 \mathrm{~b}$ & $616,20 \mathrm{~b}$ & 505,76 a \\
Margem bruta & 370,18 ab & $258,98 \mathrm{~b}$ & $1,94 \mathrm{~b}$ \\
RFD & $3,12 \mathrm{a}$ & $1,72 \mathrm{~b}$ & \\
\hline
\end{tabular}

Médias com letras distintas, na linha, diferem pelo teste de Tukey $(\mathrm{P}<0,1)$.

Preço pago pelo kg vivo novilha= R $\$ 1,50$.

$\mathrm{RFD}=$ Retorno Financeiro Direto, em R $\$$ capitalizados / R \$1,00 investido.

respostas obtidas com a aplicação de 100 e $200 \mathrm{~kg} \mathrm{ha}^{-1}$ de N. Evidentemente que, nestes dois últimos casos, aumentos em capacidade de suporte das pastagens e escala de produção devem sempre ser considerados sob o ponto de vista bioeconômico em diferentes sistemas de produção.

\section{CONCLUSÕES}

O investimento em fertilização da pastagem natural é biológica e economicamente viável, independentemente da dose de $\mathrm{N}$ até $200 \mathrm{~kg} \mathrm{ha}^{-1}$. O maior retorno direto do capital é obtido com aplicação de calcário e N-P-K de correção, sem dose de N em cobertura.

A aplicação de $200 \mathrm{~kg} \mathrm{ha}^{-1}$ de $\mathrm{N}$ em pastagem natural aumenta a produção de peso vivo, a margem bruta e a eficiência de conversão do $\mathrm{N}$ em produto animal. A resposta crescente até esta dose sugere estudos com níveis ainda superiores de $\mathrm{N}$ e queestabeleçam o real potencial produtivo deste recurso forrageiro.

\section{REFERÊNCIAS}

ANUALPEC - Anuário Estatístico da Pecuária Brasileira. São Paulo: Argos Comunicação, 2004. 400p.

AYALA, W.; CARÁMBULA, M. Nitrógeno en campo natural. In: MORÓN, A.; RISSO D.F. (Eds.). Nitrógeno en pasturas. Montevideo: INIA, 1994. p.33-42. (Série Técnica, 51).

BARCELLOS J.M. et al. Influência da adubação e sistemas de pastejo na produção de pastagens naturais. Bagé: CNPO/EMBRAPA, 1987. p.11-16. (Coletâneas das pesquisas forrageiras. V.1).

BEMHAJA, M. et al. Respuesta a la fertilización nitrogenada de campo natural en basalto profundo. In: REUNIÓN DEL
GRUPO TÉCNICO REGIONAL DEL CONE SUR EN MEJORAMIENTO Y UTILIZACIÓN DE LOS RECURSOS FORRAJEROS DEL ÁREA TROPICAL Y SUBTROPICAL: GRUPO CAMPOS, 14., 1994, Termas de Arapey. Anais... Montevideo: INIA, 1998. p.119-122. (Série Técnica, 94).

BERRETA, E.J. et al. Mejoramiento de Campo Natural de basalto fertilizado com nitrógeno y fósforo. In: SEMINARIO DE ACTUALIZACION EM TECNOLOGIAS PARA BASALTO, 1998, Tacuarembo. Anais... Montevideo: INIA, 1998. p.63-73. (Serie Tecnica, 102).

BERRETA, E.J. et al. Campos in Uruguay. In: LEMAIRE, G. et al. (Eds.). Grassland ecophysiology and grazing ecology. Wallingford: CABI, 2000. p.377-394.

BOGGIANO, P.R. et al. Efeito da adubação nitrogenada e oferta de forragem sobre as taxas de acúmulo de matéria seca numa pastagem nativa do Rio Grande do Sul. In: REUNIÃO DO GRUPO TÉCNICO EM FORRAGEIRAS DO CONE SUL ZONA CAMPOS, 18., 2000, Guarapuava. Anais... Guarapuava, 2000. p.120-121.

CARVALHO, P.C.F. et al. Importância da estrutura da pastagem na ingestão e seleção de dietas pelo animal em pastejo. In: MATTOS, W.R.S. (Ed.). A produção animal na visão dos brasileiros. SBZ: Piracicaba, 2001. p.853-871.

EMPRESA BRASILEIRA DE PESQUISA AGROPECUÁRIA EMBRAPA. Centro Nacional de Pesquisa do Solo - CNPS. Sistema Brasileiro de Classificação de Solos. Rio de Janeiro: Embrapa, 1999. 412p.

GILLEN, R.L.; BERG, W.A. Nitrogen fertilization of a native grass planting in western Oklahoma. Journal of Range Management, Lakewood, v.51, n.4, p.436-441, 1998.

GUMA, J.M.C.R. Parâmetros da pastagem e produção animal em campo nativo adubado e fertilizado com diferentes doses de nitrogênio, submetido ao diferimento para utilização no outono-inverno. 2005. 58f. Dissertação (Mestrado em Zootecnia) - Programa de Pós-graduação em Zootecnia, Faculdade de Agronomia, Universidade Federal do Rio Grande do Sul, Porto Alegre. 
MARASCHIN, G.E. A planta forrageira no sistema de produção. In: PEIXOTO, A.M. et al. (Eds.). Anais do $17^{\circ}$ Simpósio sobre manejo da pastagem. Piracicaba: FEALQ, 2000. p.285-331.

MAZZANTI, A. et al. The effect of nitrogen fertilization upon the production of tall fescue swards continuously grazed with sheep. I. Herbage growth dynamics. Grass and Forage Science, Oxford, v.49, p.111-120, 1994.

MORAES, A. et al. Comparação de métodos de taxa de crescimento em uma pastagem submetida a diferentes pressões de pastejo. In: REUNIÃO ANUAL DA SOCIEDADE BRASILEIRA DE ZOOTECNIA, 27., 1990, Campinas. Anais... Campinas: SBZ, 1990. p.332.

MORENO, J.A. Clima do Rio Grande do Sul. Porto Alegre: Secretaria da Agricultura, 1961. 41p.

MOTT, G.O.; LUCAS, H.L. The design, conduct, and interpretation of grazing trials in cultivated and improved pastures. In: INTERNATIONAL GRASSLAND CONGRESS, 6., 1952, State College. Proceedings... State College: Pensylvannia: State College, 1952. p.1380-1385.

NABINGER, C. et al. Pasture related problems in beef cattle production in southern Brazil. In: GRASSLAND
ECOPHISIOLOGY AND GRAZING ECOLOGY, 1999, Curitiba. Proceedings... Curitiba: UFPR, 1999. p.232-236.

NABINGER, C. et al. Campos in southern Brazil. In: LEMAIRE, G. et al. (Eds.). Grassland ecophysiology and grazing ecology. Wallingford: CABI, 2000. p.355-376.

PÖTTER, L. et al. Análises econômicas de modelos de produção com novilhas de corte primíparas aos dois anos, três e quatro anos de idade. Revista Brasileira de Zootecnia, Viçosa, v.29, n.3, p.861-870, 2000.

SANTOS, D.T. et al. Produção animal e retorno econômico em pastagem de milheto (Pennisetum americanum (L.) Leeke) manejada sob diferentes alturas. In: REUNIÃO ANUAL DA SOCIEDADE BRASILEIRA DE ZOOTECNIA, 40., 2003, Santa Maria. Anais... Santa Maria: SBZ, 2003. CD-Rom.

SANTOS, D.T. et al. Dinâmica de gramíneas anuais sob pastejo e doses de nitrogênio. In: REUNIÓN DE GRUPO TÉCNICO EM FORRAJERAS DEL CONO SUR, 19., 2002, Mercedes, Corrientes, Argentina. Memorias... Mercedes: INTA, 2002. p.177-178.

WHITEHEAD, W.C. Grassland nitrogen. Wallingford: CAB International, 1995. 397p. 